\title{
Ongoing CNES space optics developments
}

\section{Caroline Laurent}

Caroline Laurent, "Ongoing CNES space optics developments," Proc. SPIE 11852, International Conference on Space Optics - ICSO 2020, 1185202 (11 June 2021); doi: 10.1117/12.2599142

SPIE Event: International Conference on Space Optics - ICSO 2021, 2021, Online SPIE. Only 


\section{International Conference on Space Optics-ICSO 2020}

Virtual Conference

30 March-2 April 2021

Edited by Bruno Cugny, Zoran Sodnik, and Nikos Karafolas
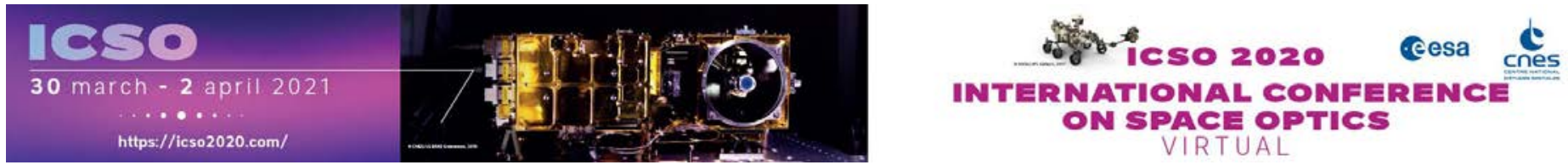

\section{Ongoing CNES space optics developments}

\section{Cesa isopmeatians ecnes}

International Conference on Space Optics - ICSO 2020, edited by Bruno Cugny, Zoran Sodnik, Nikos Karafolas, Proc. of SPIE Vol. 11852, 1185202 - (c) 2021 ESA and CNES

CCC code: $0277-786 \mathrm{X} / 21 / \$ 21 \cdot$ doi: $10.1117 / 12.2599142$ 


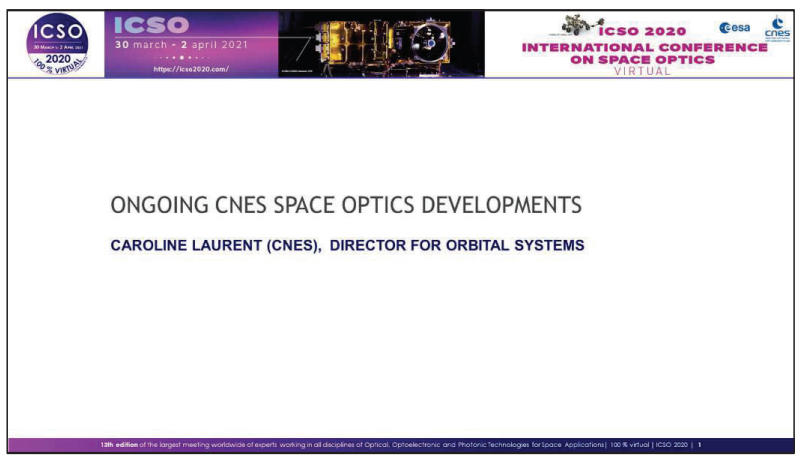

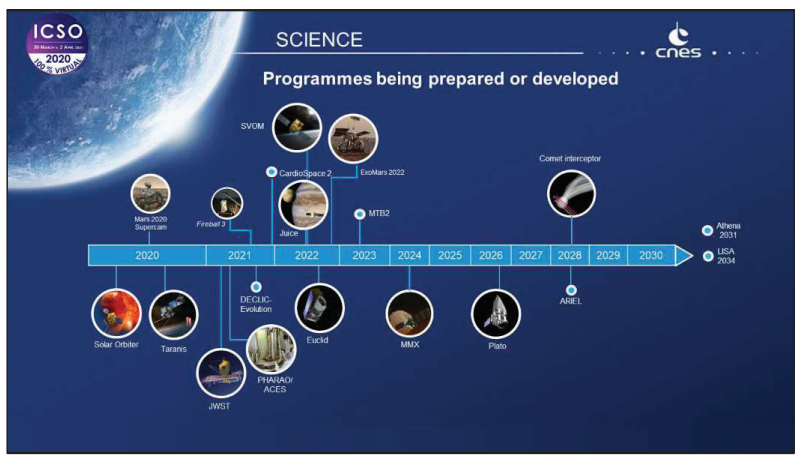

Most of french contributions to Science programmes are involved in the framework of ESA Cosmic Vision programme. Beside these european missions, CNES has developed bilateral or multilateral cooperations with other space agencies. Let us make a focus on 4 of them : Supercam, SVOM, MMX and X-IFU/Athena

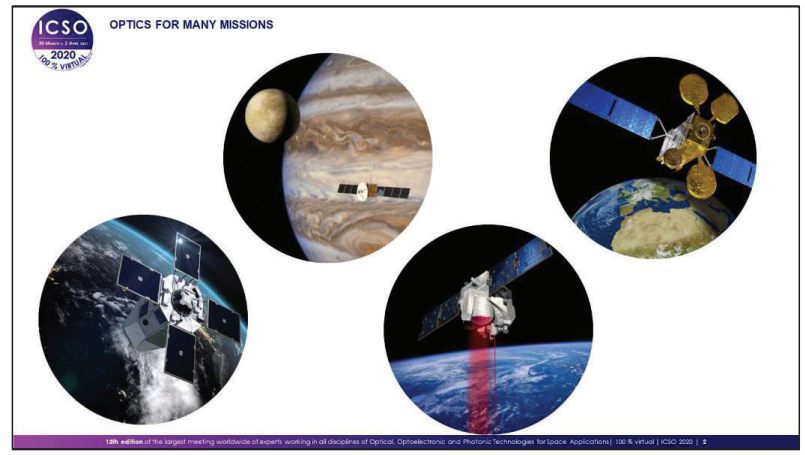

CNES has been developing space programs for decades in four principal domains : Science / Exploration, Defense, Earth Observation, and Telecommunications (we put aside the launcher domain). All of them involve optical systems, mainly as the core payload for instrumentation, or as a technology for laser links.

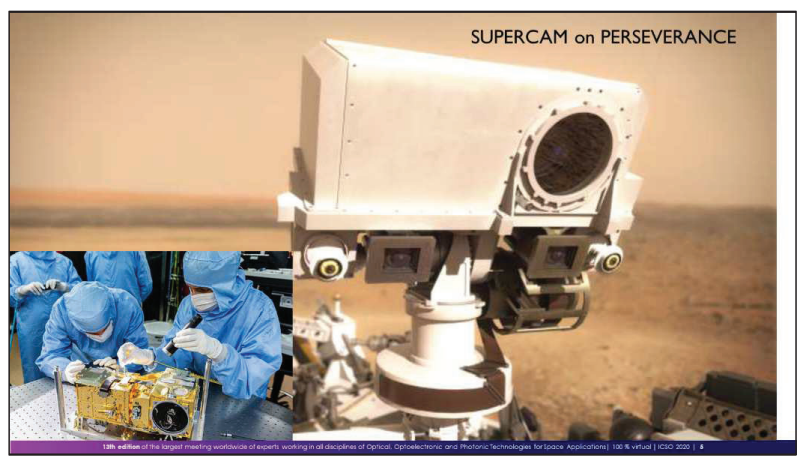

In spite of a major accident during the development, its delivery on time to JPL has been one of the most challenging and successfull milestone, thanks to the efforts and passion of the whole project team. You can see some of them at IRAP premises, and I

recommend to attend the fascinating talk of Pernelle Bernardi (LESIA) on SuperCam LIBS (session 15 on Wednesday).

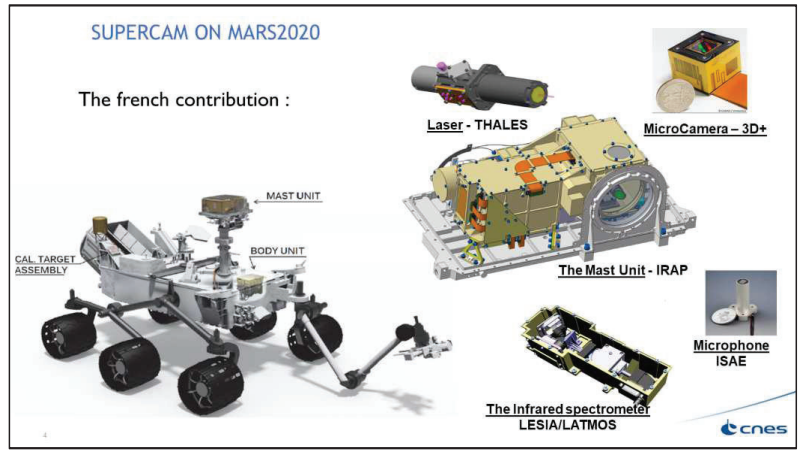

Supercam has been in the spotlight since Perseverance landed on Mars. This french contribution inherits directly from Chem Cam on Curiosity but with many significant improvements like Raman and infrared spectroscopy, and coloured imagery.

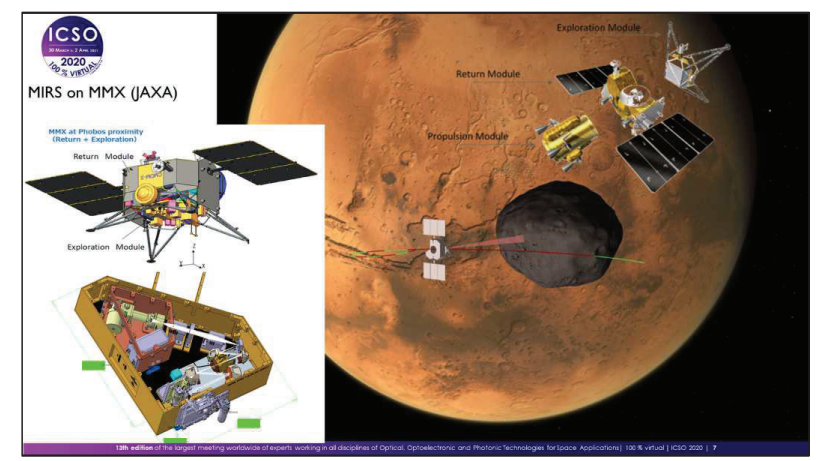

MMX (Martian Moons Exploration) developped by JAXA (+ contributions from other agencies) aims to explore Mars and its moons and bring back Phobos samples (Phobos Grunt unfortunately failed in 2011)

Among many other sensors MIRS is a compact (less than $12 \mathrm{~kg}$ and as big as a shoe box) IR imaging spectrometer codesigned by CNES and french laboratories (LESIA...) on the Exploration module. 
ICSO 2020

Virtual Conference

International Conference on Space Optics
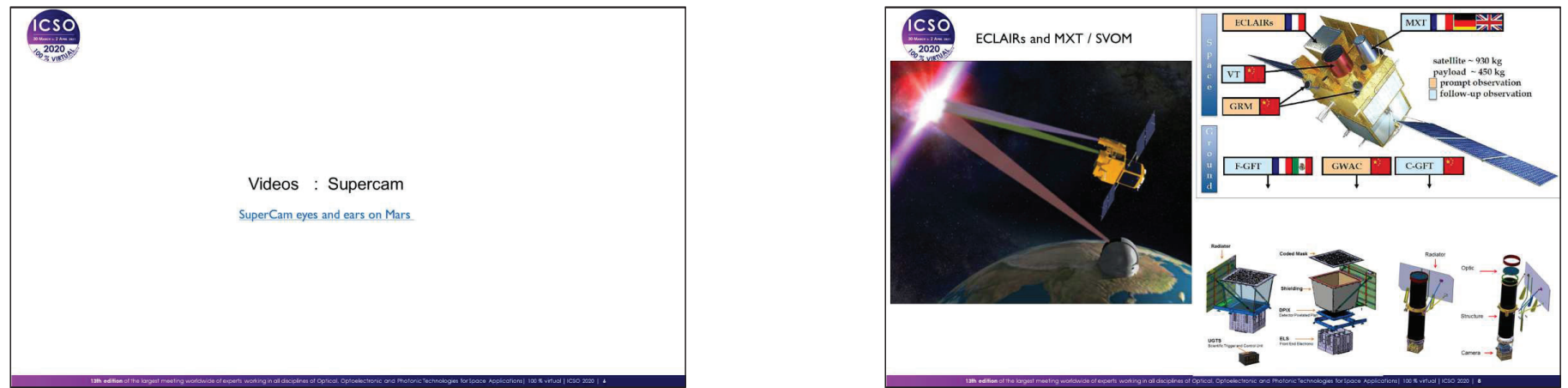

SVOM (Space Variable Objects Monitor) is an astrophysics mission whose main objective is to observe and characterise gamma-ray bursts, the most powerful phenomena in the Universe. The mission, the satellite and the platform are under Chinese responsibility while the instruments and the ground segment are shared between China and France.

The French contribution consists of ECLAIRs and MXT instruments (Gamma Ray and $X$ ray Telescopes), as well as the French parts of the ground segment (ECLAIRs and MXT control centres, data processing centre, network of alert antennas).
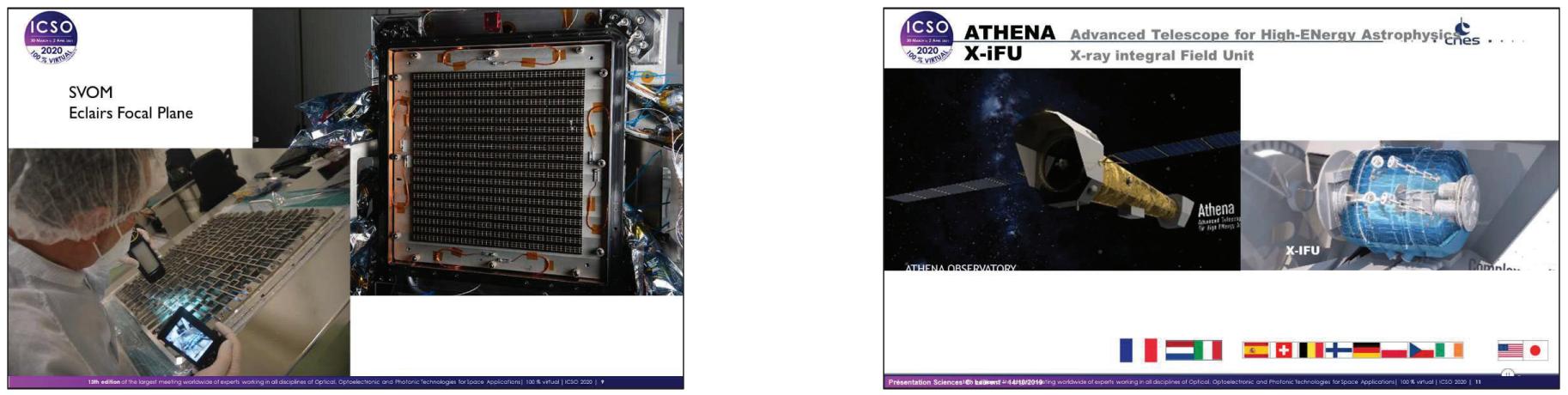

ECLAIRs : is a X-/Gamma-ray wide-field $\left(89^{\circ} \times 89^{\circ}\right)$ coded-mask camera working in the 4-250 keV energy range. View of the coded Mask (left) and DPIX (right) focal plane composed of $6400 \mathrm{CdTe}$ detectors working around $-20^{\circ} \mathrm{C}$

The ECLAIRs consortium is made up of several French labs: IRAP, IRFU \& APC. The ECLAIRs telescope is under the CNES supervision.

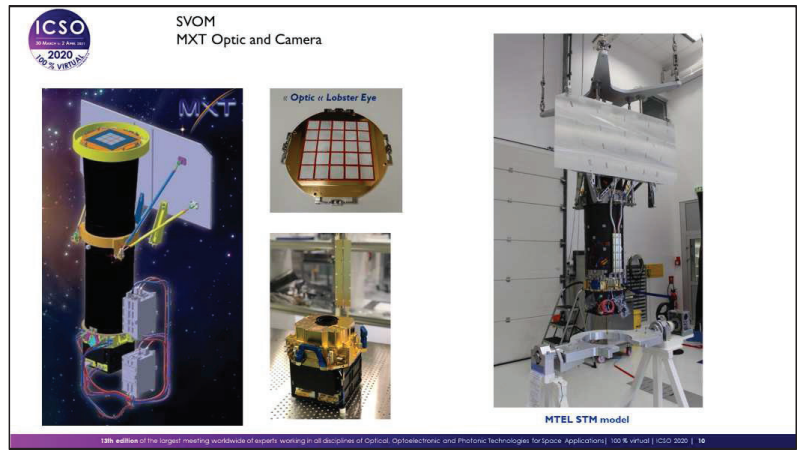

Athena X-ray Observatory; the second large mission of the European Space Agency science program, to be launched in the early 2030s. The Athena mission is designed to implement the Hot and Energetic Universe science theme. $X$-IFU is an Integrat Field Unit at the focal plane of a huge and novelX Ray telescope $X$-I U is an ESA during countries are in charge of the development of this huge instrument $(800 \mathrm{~kg} / 3000 \mathrm{~W})$ The X-IFU is a cryogenic X-ray spectrometer, based on a large array of Transition Edge Sensors (TES), offering $2.5 \mathrm{eV}$ spectral resolution, with 5 arcsecond pixels, over a field of view of 5 arc minutes in equivalent diameter.

Around 3200 detectors (Microcalorimeters) to measure the amount of heat released when an X-Ray is absorbed

cooled to nearly absolute zero $(50 \mathrm{mK})$.

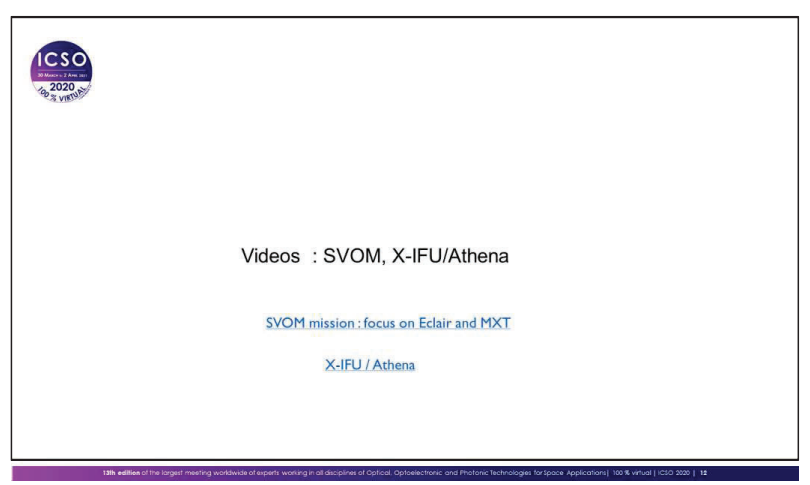

MXT (Microchannel X-ray Telescope)

In response to alerts transmitted by ECLAIRs, the Microchannel X-ray Telescope (MXT) will observe gamma ray bursts (GRBs) in the soft X-ray range (energy between 0.2 and $10 \mathrm{KeV}$ ), from the very beginning of their afterglow emission. MXT is being developed in France by CNES and CEA/Irfu, in close collaboration with the University of Leicester in the United Kingdom and the Max-Planck Institute für extraterrestische Physik in Germany. 


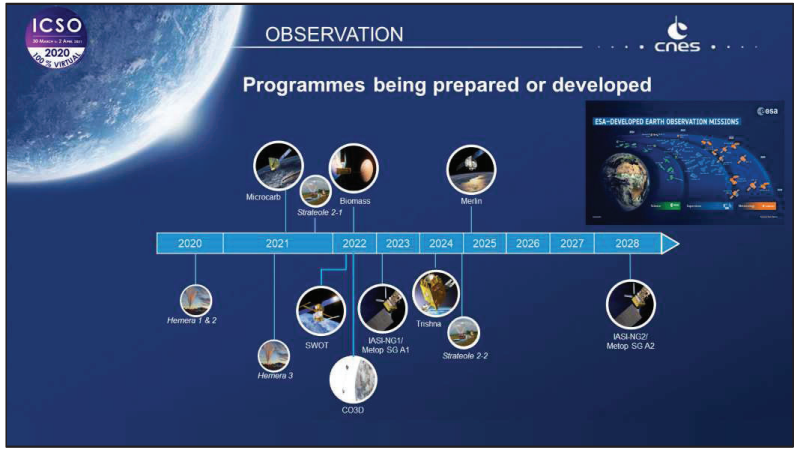

Of course, many french contributions to ESA developped EO missions as presented on the right (Earth Explorer, Copernicus and recent HPCM Copernicus expansion missions, Eumetsat misions). In parallel, CNES develops bilateral or multilateral cooperations with other space agencies. Let us make a focus on three of them : IASI-NG, Microcarb, Trishna and a more prospective one on Quantum Gravimetry

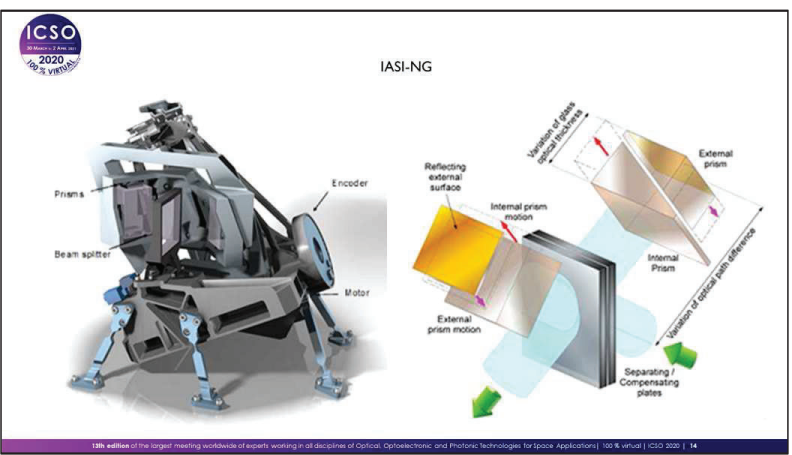

IASI-NG is the follow-on of the successfull IASI instrument developped by TAS for METOP. Performances requirements improvements (X2 spectral and radiometric resolutions) have led to a new concept proposed by ADS (Interferometer with field compensation and qualified $\mathrm{KBr}$ for prism and beam-splitter)

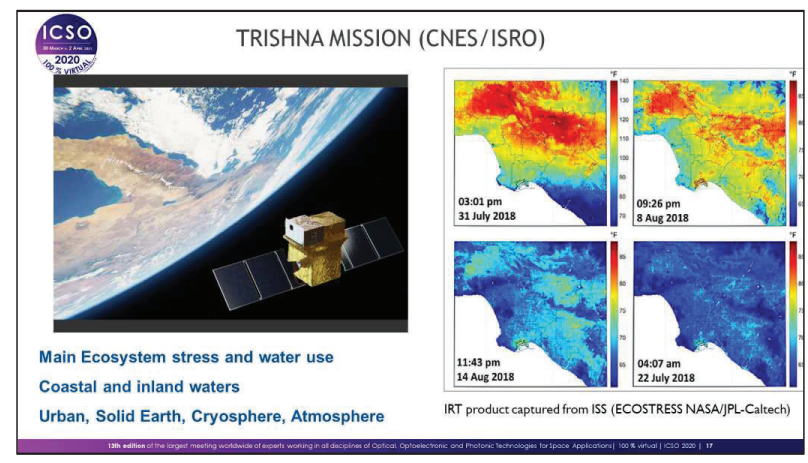

The TRISHNA mission (Thermal infraRed Imaging Satellite for High-resolution Natural resource Assessment) is a cooperation between CNES and Indian (ISRO) space agencies. It is intended to measure approximately twice a week the thermal infrared signal of the surface-atmosphere system globally and at $57 \mathrm{~m}$ resolution for the continents and the coastal ocean, and a resolution of $1000 \mathrm{~m}$ over deep ocean.

The scientific objectives are the monitoring of the water status of continental ecosystems and the monitoring of coastal and continental waters. Additional themes are the study of Urban Environments, Cryosphere, Atmosphere and Solid Earth.

ECOSTRESS imagery shows surface temperature variations in Los Angeles, California between July 22 and August 14 at different times of day. Hot areas are shown in red, warm areas in orange and yellow, and cooler areas in blue (image credit: NASA/JPL-Caltech)

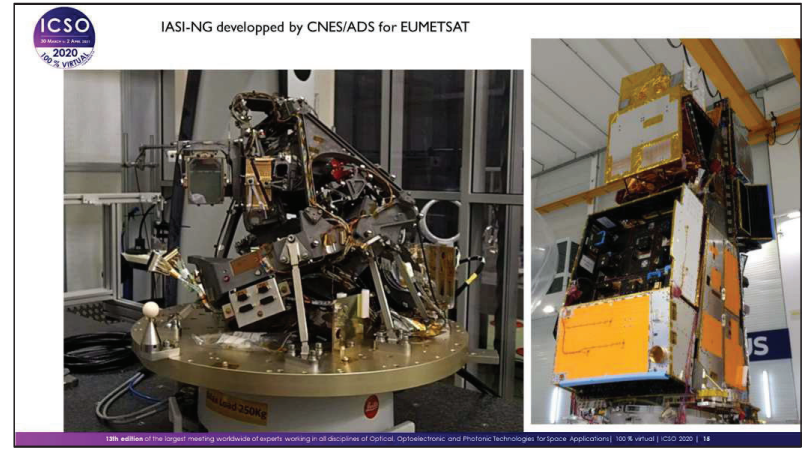

From concept to reality: IASI-NG optical Mertz interferometer developped by ADS and IASI-NG STM at the top of METOP-NG satellite. See tomorrow morning Adrien Deschamps plenary dedicated to IASI as well as technical talks from CNES and ADS Thursday afternoon in session 38

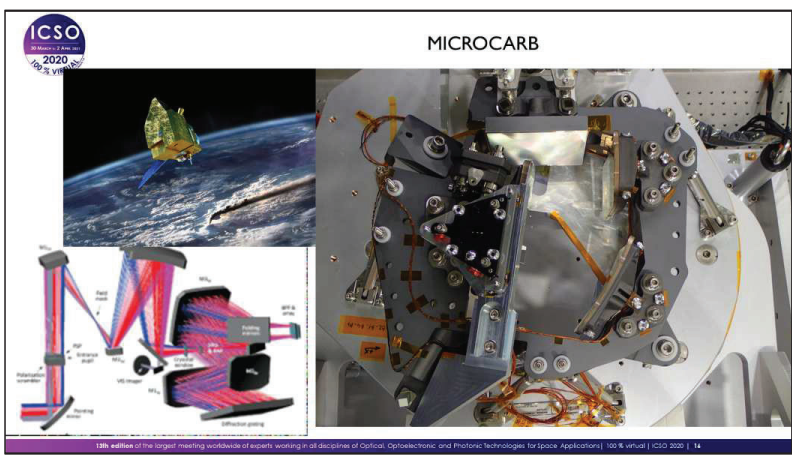

MicroCarb will monitor the fluxes of carbon dioxide in order to improve our knowledge on the carbon cycle. The camera takes advantage of an original concept to measure the short-wave infrared light spectrum in 4 bands on the same detector. Free-form optics are used to make the camera as compact as possible.

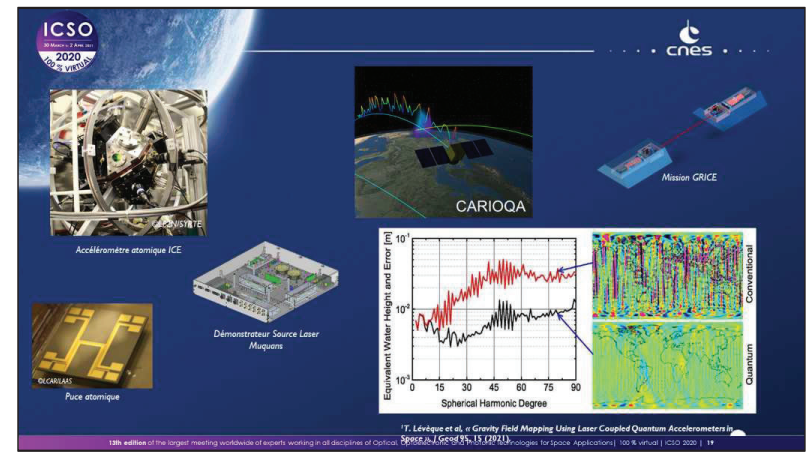

Cold atoms experiments have started in CNES more than 10 years ago through severa french collaborations (CNES R\&D, ONERA, SYRTE, LP2N, LCAR, Muquans). It was illustrated in 2014-2016 by the Zero G airborne demonstration ICE (Interférométrie atomique à sources Cohérentes pour l'Espace / Equivalence principle at atomic level) and the first cold atom interferometric fringes (Nature publication). Works have been recently oriented towards high performances atomic accelerometer and a proposa of an in-orbit demonstration (pathfinder) named CARIOQA to prepare the follow-on of the historic serie of international geodesy missions (CHAMP, GRACE, GOCE, GRACE-FO, GRACE II (US) NGGM (ESA)) for an improved spatial and temporal resolution (10 days, below $100 \mathrm{~km})$. 


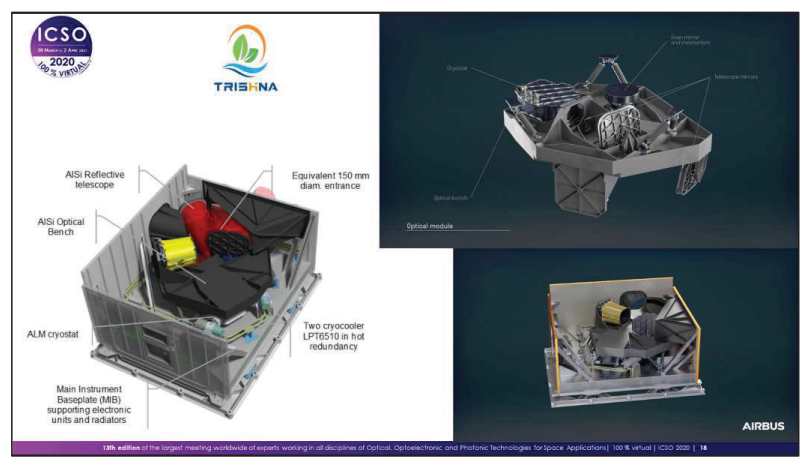

The payload is composed of a VIS/SWIR instrument (ISRO) and a TIR instrument (CNES) shown on the slide with 4 TIR bands from 8,6 to 11.6 microns.

Development status of Trishna instrumentation will be presented by CNES/ADS this afternoon in session 6 (paper 267)

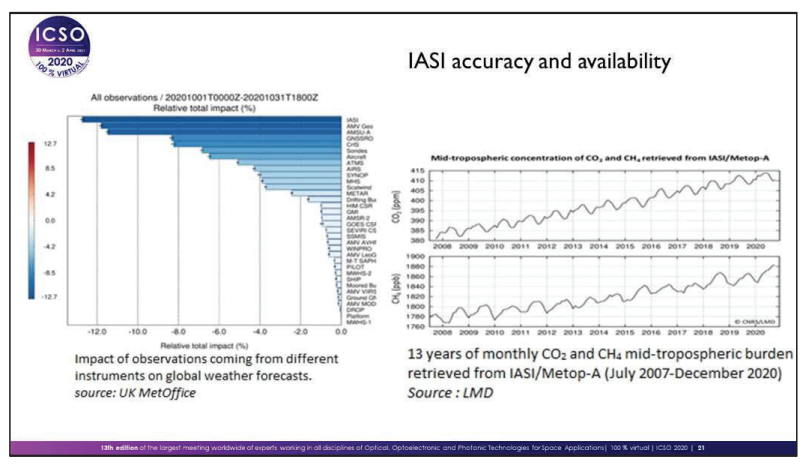

Excellence in sounding instrumentation is an achievement and we can be proud to share that IASI results have the first rank for global weather forecast. Beside its operational use by weather forecast agencies, IASI shows very consistent scientifical results that contribute to a better knowledge for our future climate.

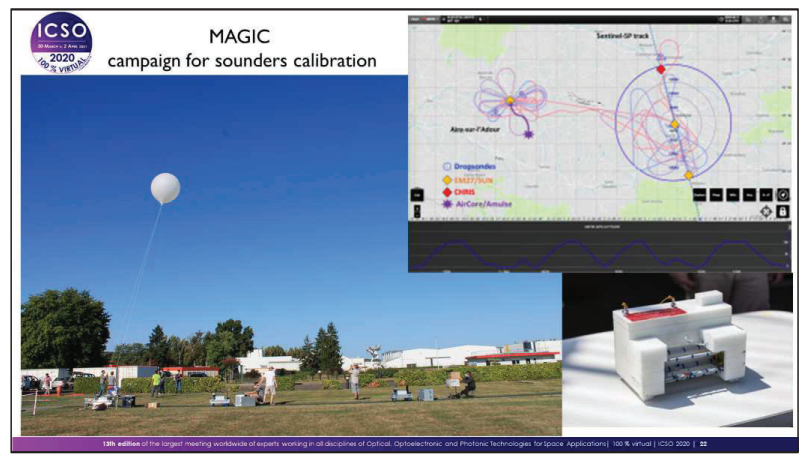

In order to improve the calibration process and the errors budget for future greenhouse gaz missions like IASI-NG, MicroCarb or Merlin, CNES has undertaken specific calibration campaigns with its partners. Thanks to AirCore from balloons and spectrometers from planes, atmosphere profiles can be used as references and cross-calibration with satellites that overpass.

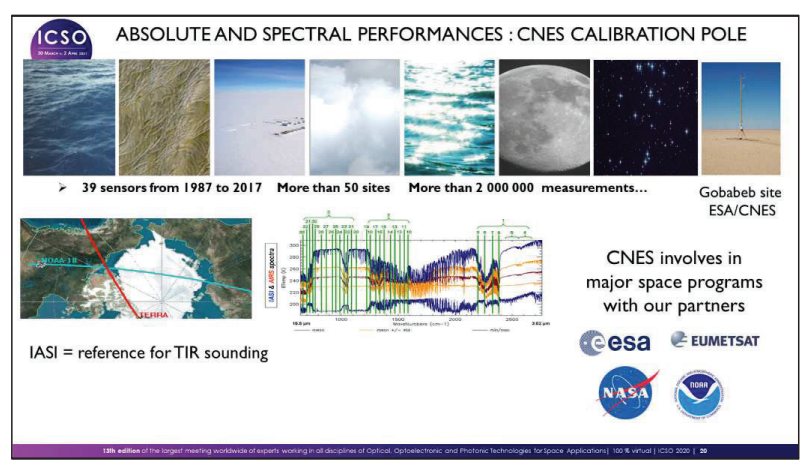

As an agency, CNES has contributed to the global remote sensing community with its comprehensive toolbox of calibration methods and huge database of sensors and sites measurements, from multispectral imagery to thousands of bands in IASI infrared atmospheric spectrum
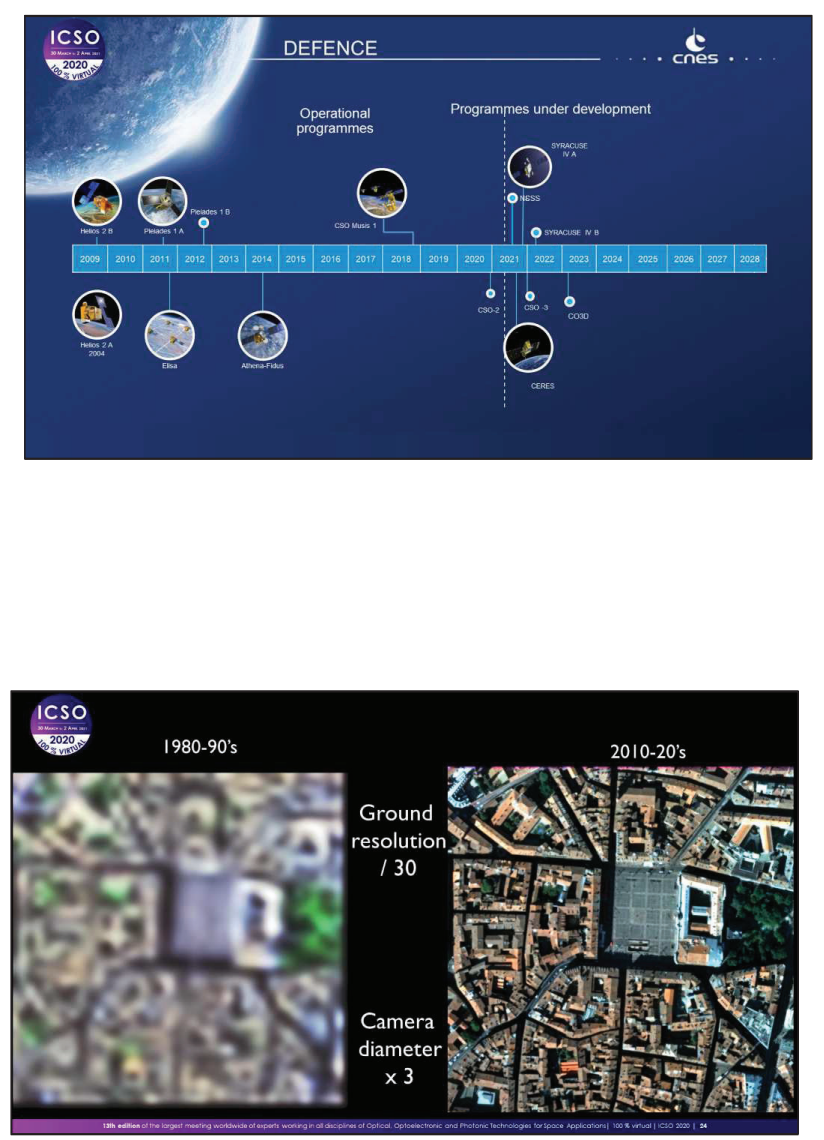

Satellite imagery resolution has been dramatically improved for 30 years. Commercial imagery is now available at very high resolution once restricted to defence use. This improvment unveils a significant effort to get closer and closer to the optics cut-off frequency, which means one order of magnitude for the product Resolution $\mathrm{x}$ diameter. 


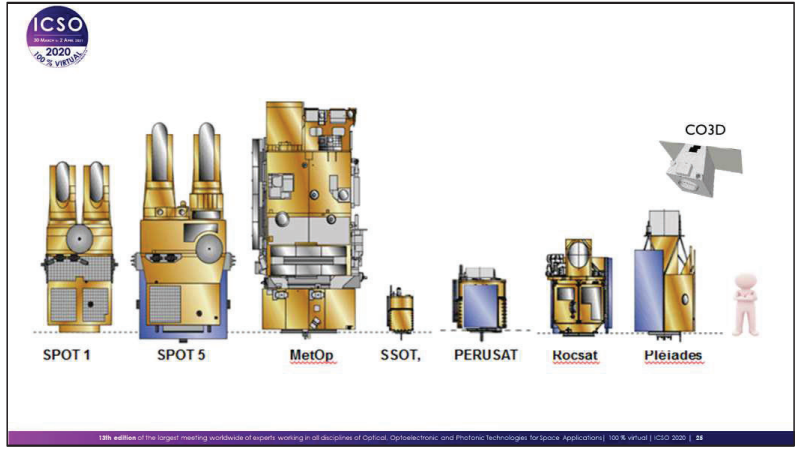

Making small cameras is the main cost-effective driver for affordable satellites. The imaging programmes developed by our prime contractors for export or commercial purposes have set a global benchmark.

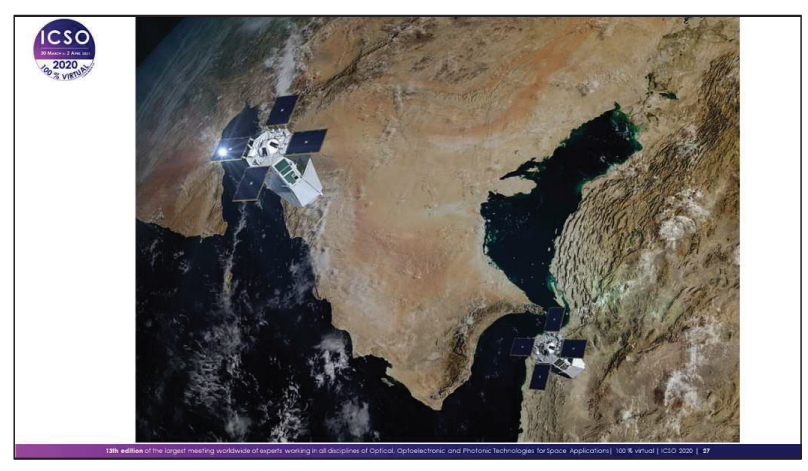

France has played a leading role on very high resolution optical imagery for 30 years. We keep on improving the operational capabilities for defence users, and can be very proud of the performances reached by the CSO-2 satellite since its recent launch. This success was possible thanks to the efforts of DGA, CNES and our industry that masters a very thorough know-how in optical high technologies.

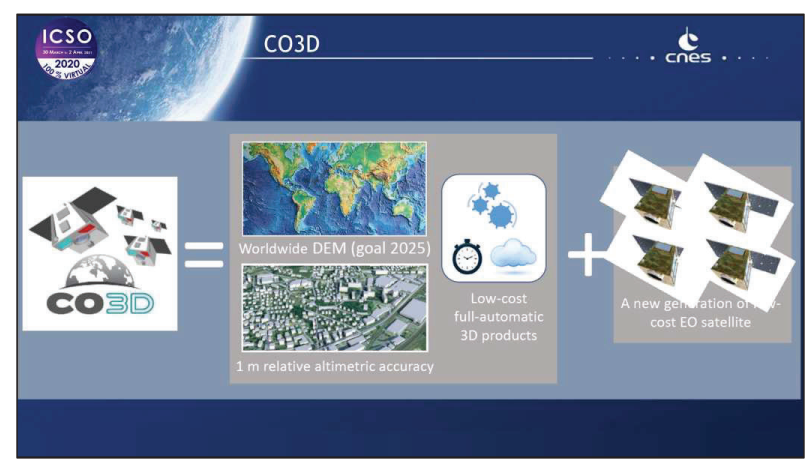

The CO3D programme is an innovative programme in partnership between CNES and $A D S$, in order to develop 4 identical imaging satellites that operate to acquire accurate stereoscopic imagery of the Earth with a $50 \mathrm{~cm}$ ground resolution. The system will be able to produce and update a global DTM each year. The camera and the satellite are designed to be ready for export and larger constellations.

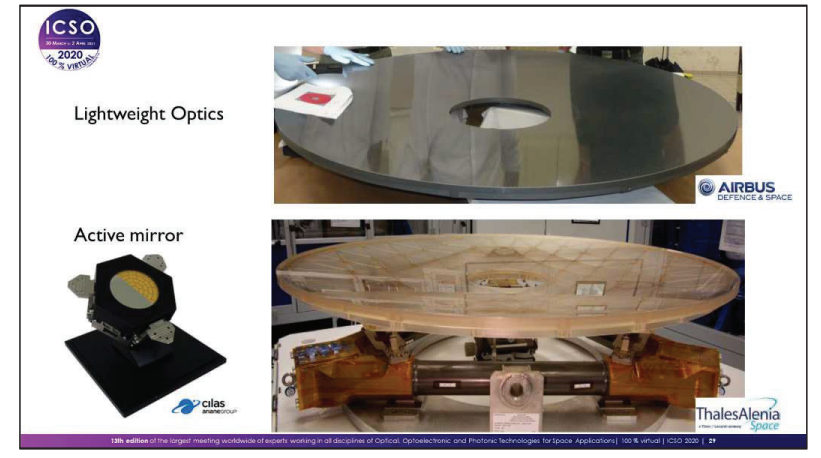

Even if optical cameras are fully optimized, future very high resolution satellites need very large optics that has to be very light. CNES and DGA have led thorough studies with our main manufacturers in order to qualify large lightweight mirrors, on-board metrology, and active mirrors to compensate for optical deformations.

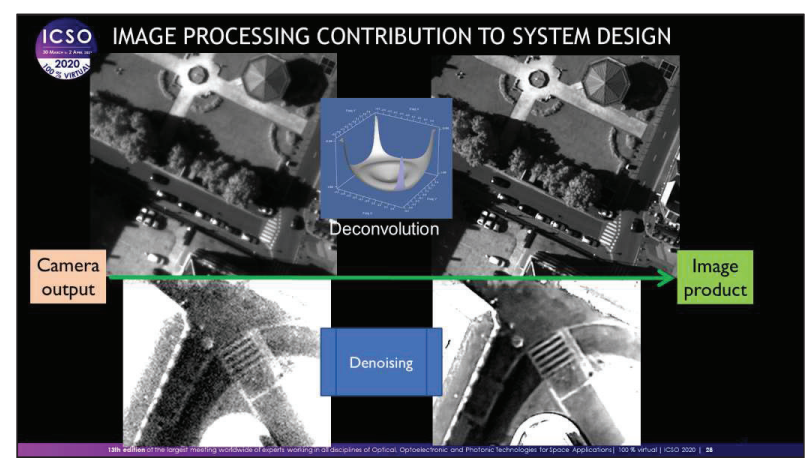

Image restoration is a key to design affordable high resolution cameras and satellites : fuzzy and noisy raw images can be deconvolved and denoised. This image processing capability is the main tool not to oversize the mirror size. These technics are very helpful in the framework of high resolution (either spatial or spectral) where the useful signal may be very low

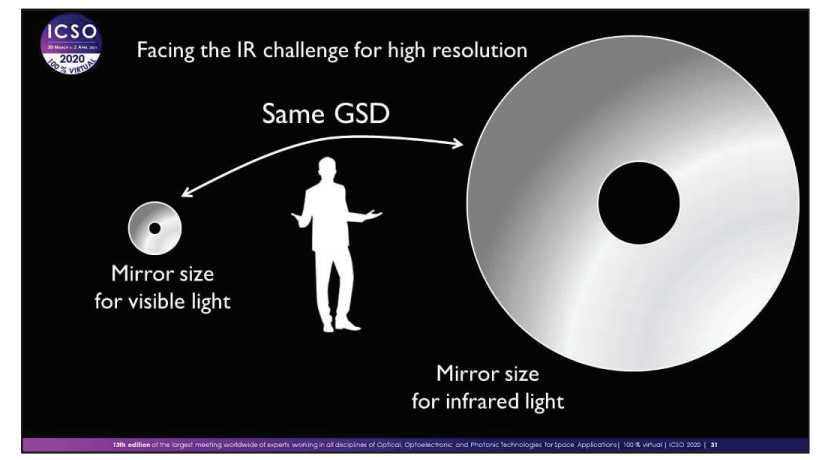

Night-time imagery from space implies either radar or infrared imagery. The latter is a challenge because infrared wavelength is 6 to 12 times larger than visible wavelength, and the mirror size is proportional to the wavelength. That's why it is a long way to be able to get very high resolution infra-red imagery. 


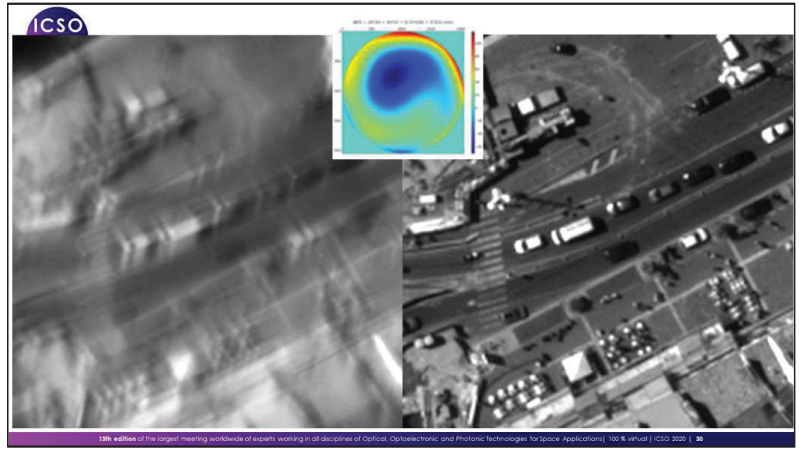

Next generation very high resolution satellites need large and light optics which shape may vary in orbit due to gravity and thermal conditions. CNES has carried out thorough studies to adapt active optics technics from astronomy to imagery satellites.

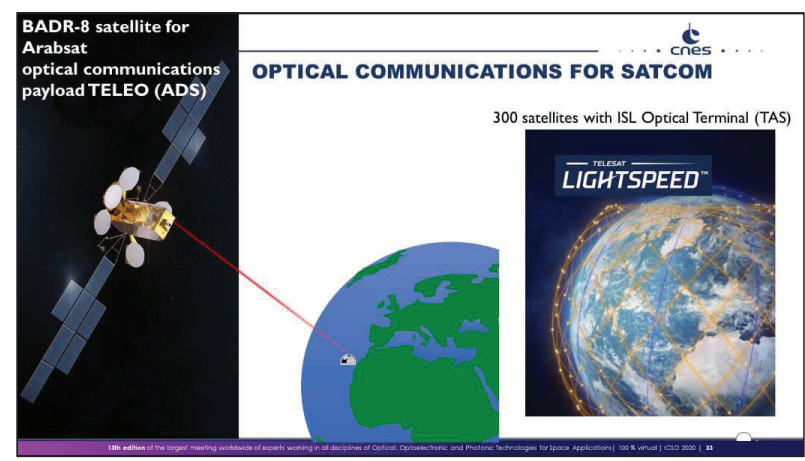

Very recently ADS has announced the development of TELEO, an optical communication payload on BADR-8 Geo satellite (Feederlink performance assessment) and TAS has been selected as MOI for next LIGHTSPEED constellation (Telesat) of 298 Low earth orbit satellites with 4 optical terminals per satellite

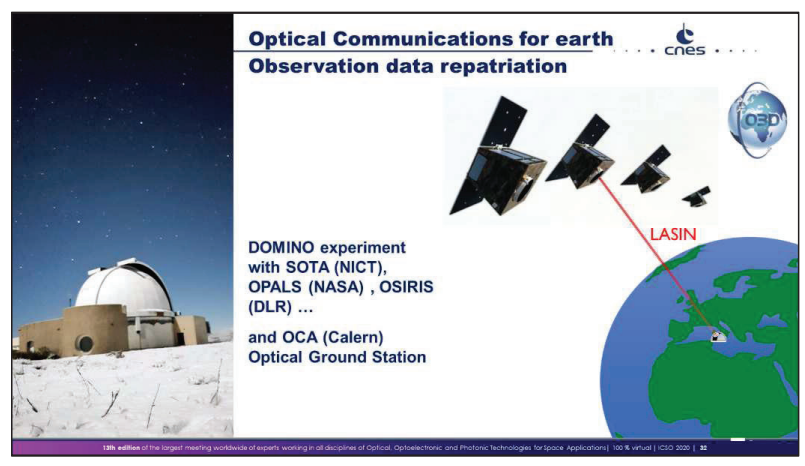

For several years CNES has developped DOMINO experiment (with ONERA and OCA supports) based on opportunity satellites operated by differents agencies (NICT, NASA, DLR ...) and the OCA optical ground station (CALERN). The objective was to assess with cooperative emitters telemetry performances with representative atmospheric conditions and until low incidence angles. The LASIN "LASer through INstrument " embarked on CO3D aims to demonstrate the data repatriation performances from low earth orbit. The agility of the CO3D platform is used to target the Optical Ground station.

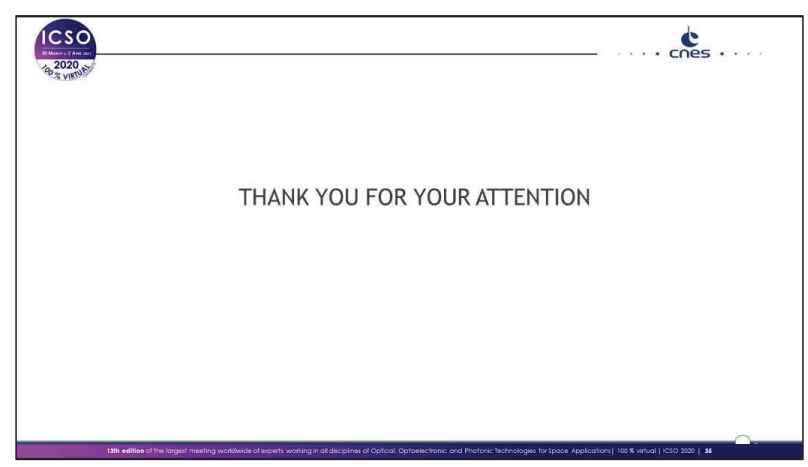

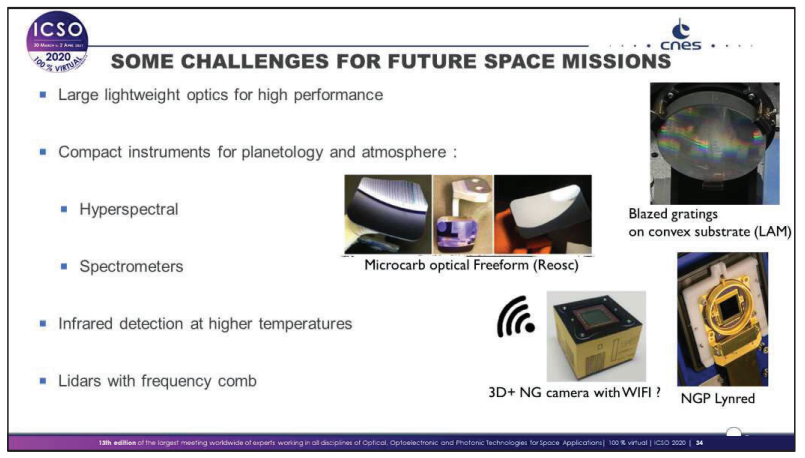

For the future, continuation of CNES efforts (Labs \& Industry with R\&D, Thesis, Proof of Concept and Higher TRL level demonstrators) :

-Prepare high performance lightweight optics (Very High Angular resolution prepared by OTOS/TANGO) see paper 218 by TAS

-Miniaturization of high performances instruments (see Microcarb) with use of new materials, free-form optics, specific gratings or linear filter on focal plane, additive manufacturing ...

Hyperspectral instrumentation, new spectrometers concepts for constellation of smallsats dedicated to atmospheric measurements

-Use of available power processing inside CMOS Detectors (Cube 3D+NG) for AI algorithms dedicated to new applications (ex : monitoring of very wide field of view)

-Monitor technological developments on CMOS (colour, polarization, ...) and IR detectors (SWIR in particular not covered by Defense) at higher temperature -Lidar : Monitor technological developments on the most critical technologies (power lasers, freq comb, low flux detectors, specific optical filters ...) and development of functional breadboards to assess performances and identify critical trade off of new concepts (SCALE) 